Correction

\title{
Correction: Combining molecular and immunohistochemical analyses of key drivers in primary melanomas: interplay between germline and somatic variations
}

\author{
William Bruno ${ }^{1}$, Claudia Martinuzzi ${ }^{1}$, Bruna Dalmasso ${ }^{1}$, Virginia Andreotti ${ }^{1}$, \\ Lorenza Pastorino1, Francesco Cabiddu2, Marina Gualco², Francesco Spagnolo ${ }^{3}$, \\ Alberto Ballestrero ${ }^{1}$, Paola Queirolo ${ }^{3}$, Federica Grillo ${ }^{4,}$, Luca Mastracci,,* and \\ Paola Ghiorzo ${ }^{1, *}$ \\ ${ }^{1}$ Department of Internal Medicine and Medical Specialties (DiMI), University of Genoa and Ospedale Policlinico San Martino, \\ Genoa, Italy \\ 2 Pathology Unit, Ospedale Policlinico San Martino, Genoa, Italy \\ 3 Department of Medical Oncology, Ospedale Policlinico San Martino, Genoa, Italy \\ ${ }^{4}$ Department of Surgical and Diagnostic Sciences, Pathology Unit, University of Genoa and Ospedale Policlinico San Martino, \\ Genoa, Italy \\ * These authors contributed equally to this work \\ Published: June 19, 2018
}

Copyright: Bruno et al. This is an open-access article distributed under the terms of the Creative Commons Attribution License 3.0 (CC BY 3.0), which permits unrestricted use, distribution, and reproduction in any medium, provided the original author and source are credited.

This article has been corrected: The proper listing of author names is given below:

William Bruno ${ }^{1}$, Claudia Martinuzzi ${ }^{1}$, Bruna Dalmasso ${ }^{1}$, Virginia Andreotti ${ }^{1}$, Lorenza Pastorino1, Francesco Cabiddu², Marina Gualcoㄹ, Francesco Spagnolo ${ }^{3}$, Alberto Ballestrero ${ }^{1}$, Paola Queirolo ${ }^{3}$, Federica Grillo ${ }^{4, *}$, Luca Mastracci,,* and Paola Ghiorzo ${ }^{1, *}$

Original article: Oncotarget. 2018; 9:5691-5702. https://doi.org/10.18632/oncotarget.23204 